\title{
Chlorine Promotion of Styrene Epoxidation on Au(111)
}

\author{
Dilini S. Pinnaduwage, Ling Zhou, Weiwei Gao, Cynthia M. Friend*
}

\section{Supporting Information}

\section{Experimental Section:}

All experiments were conducted in an ultra-high vacuum (UHV) chamber with a base pressure of $\sim 1 \times 10^{-9}$ Torr, which is equipped with a quadrupole mass spectrometer (Pfeiffer, QMS 200) and an X-ray photoelectron spectrometer (PHI ESCA 5300). For temperature-programmed reaction spectroscopy (TPRS) and temperature-programmed desorption spectroscopy (TDS), the sample was moved close to the QMS ionizer, and the heating wire was degassed before the reaction, in order to ensure that only those species desorbing from the crystal surface contribute to the spectra. The heating rate was $\sim 10$ $\mathrm{K} / \mathrm{s}$. Scanning tunneling microscopy (STM) experiments were conducted in a UHV chamber with a base pressure of $\sim 1 \times 10^{-9}$ Torr. This system is equipped with a homemade 'beetle-type' STM and auger electron spectroscopy (AES).

The $\mathrm{Au}(111)$ single crystal was radiatively heated by the tungsten filament behind the sample. The temperature was monitored by means of a C-type thermocouple press-fit into a hole on the side of the crystal. The $\mathrm{Au}(111)$ surface was cleaned by repeated cycles of $\mathrm{Ar}^{+}$sputtering $(1000 \mathrm{eV}, 1 \mu \mathrm{A})$ at $300 \mathrm{~K}$ followed by annealing at $900 \mathrm{~K}$ for 5 minutes and a slow annealing at $700 \mathrm{~K}$ for 1 hour until no impurities were detected by XPS.

The O-covered Au(111) surface was prepared by ozone exposure, as described in detail elsewhere ${ }^{1}$. Oxygen coverage was determined by the comparison of the integrated 
area of the $\mathrm{O}_{2}$ peak obtained in TPD. The saturation oxygen coverage was taken to be $\sim$ 1 ML based on previous calibration studies ${ }^{1,2}$. Chlorine coverage was determined by comparing the integrated area of the $\mathrm{Cl}_{2}(\mathrm{~m} / \mathrm{z}=70)$ desorption peak obtained in TPD. The saturation chlorine coverage was taken to be $\sim 2.9 \mathrm{ML}$ based on the previous study of $\mathrm{Cl}_{2}$ adsorption on $\mathrm{Au}(111)^{3}$. Styrene (Alfa Aesar, 99.5\%) was stored in a glass dosing tube attached to the UHV chamber through a leak valve and was purified by repeated freezepump-thaw cycles. Styrene was introduced to the surface by direct dosing through a 5 $\mathrm{mm}$ diameter tube ending about $10 \mathrm{~mm}$ from the sample surface.

Isothermal styrene reactions at $300 \mathrm{~K}$ were conducted by first dosing ozone on clean $\mathrm{Au}(111)$ at $300 \mathrm{~K}$ to obtain $\sim 0.3 \mathrm{ML}$ of oxygen followed by background dosing of styrene at $1 \times 10^{-9}$ Torr at $300 \mathrm{~K}$ with the crystal facing the Mass Spectrometer. The same procedure was followed for the chlorine promoted isothermal styrene reactions with $\sim 1.2$ ML of chlorine pre-dosed on the Au surface at $300 \mathrm{~K}$. 


\section{Styrene epoxide product identification:}

Table 1. Mass spectra fragmentation patterns for styrene oxide from the reaction of styrene with oxygen on $\mathrm{Au}(111)$, reaction of styrene with oxygen on chlorine pre-dosed $\mathrm{Au}(111)$, and for selected samples with stoichiometry $\mathrm{C}_{8} \mathrm{H}_{8} \mathrm{O}$. The absence of $\mathrm{m} / \mathrm{z}=119$ in geometric isomers of styrene oxide confirms the formation of styrene oxide.

\begin{tabular}{|l|c|c|c|c|c|}
\hline \multirow{2}{*}{ Sample } & \multicolumn{5}{|c|}{ mass/charge ratio (intensity) } \\
\cline { 2 - 6 } & $\mathbf{9 1}$ & $\mathbf{9 2}$ & $\mathbf{1 1 8}$ & $\mathbf{1 1 9}$ & $\mathbf{1 2 0}$ \\
\hline $\begin{array}{l}\text { Styrene Oxide product } \\
\text { O/Au on }\end{array}$ & 100 & 28 & 0 & 50 & 91 \\
\hline $\begin{array}{l}\text { Styrene Oxide product } \\
\text { O/C1/Au on }\end{array}$ & 100 & 31 & 0 & 47 & 81 \\
\hline Styrene Oxide & & & & & 71 \\
\hline Styrene Oxide $^{\mathbf{c}}$ & 100 & 26 & 0 & 50 & $(36)$ \\
\hline Phenylacetaldehyde $^{\mathbf{b}}$ & $(100)$ & $(29)$ & $(0)$ & $(21)$ & $(36)$ \\
\hline Phenylacetaldehyde $^{\mathbf{c}}$ & $(100$ & 26 & 0 & 0 & 25 \\
\hline Acetophenone $^{\mathbf{c}}$ & $(6)$ & $(0)$ & $(0)$ & $(0)$ & $(100)$ \\
\hline
\end{tabular}

${ }^{\mathrm{a}}$ Fragmentation patterns measured for the reaction products at $310 \mathrm{~K}$ for styrene on $\mathrm{O} / \mathrm{Au}$ and $240 \mathrm{~K}$ for styrene on $\mathrm{O} / \mathrm{Cl} / \mathrm{Au} .{ }^{b} \mathrm{Fragmentation}$ patterns measured for the molecular desorption peaks from clean $\mathrm{Au}(111) .{ }^{\mathrm{c}}$ Values in parentheses are reference data from the NIST database. 


\section{TPD spectra for O/Au(111) and O/Cl/Au(111):}

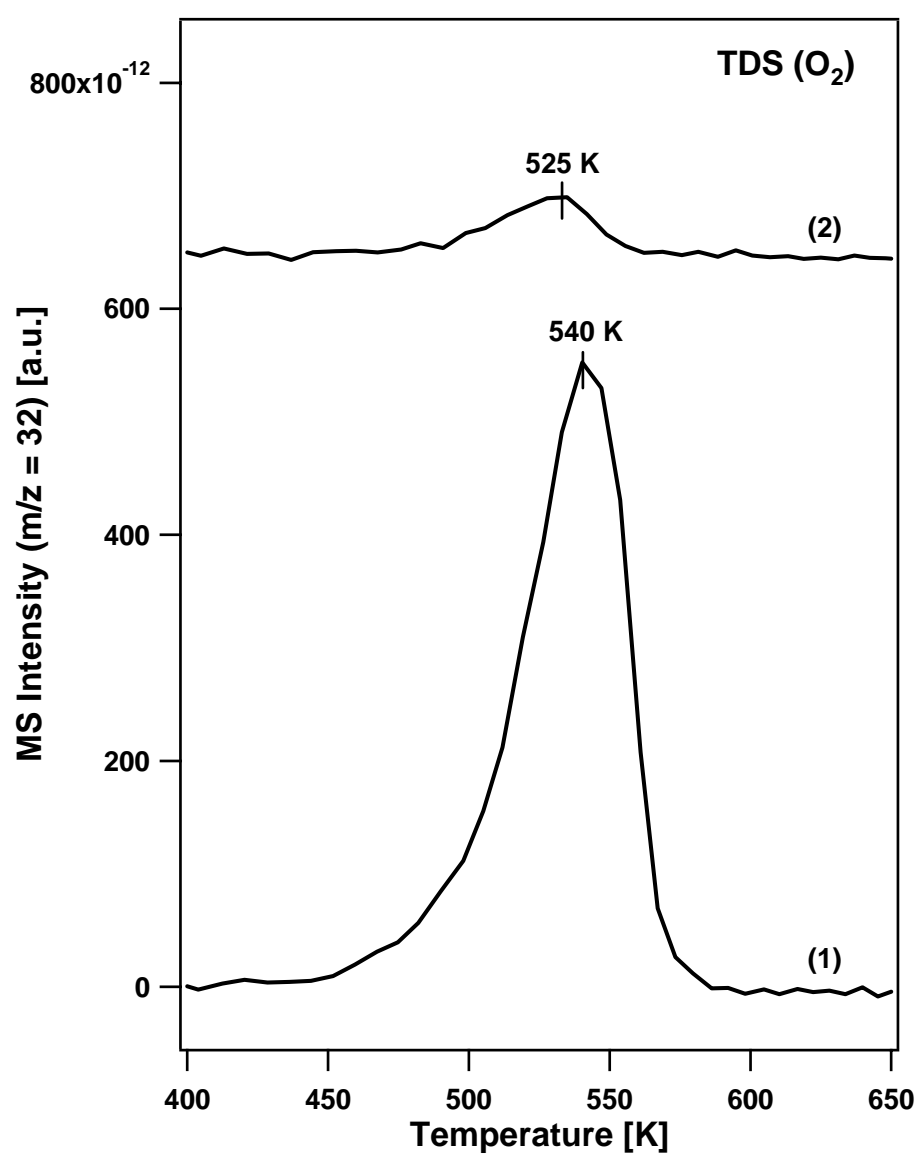

Figure S1. (a) Temperature-programmed desorption spectra $(\mathrm{m} / \mathrm{z}=32)$ for (1) $0.3 \mathrm{ML}$ oxygen-covered $\mathrm{Au}(111)$ and (2) $\mathrm{Au}(111)$ with 1.2 ML pre-dosed chlorine followed by the same ozone dosage as in (1). 


\section{Mass 44 TPD for post-oxidation reactions:}

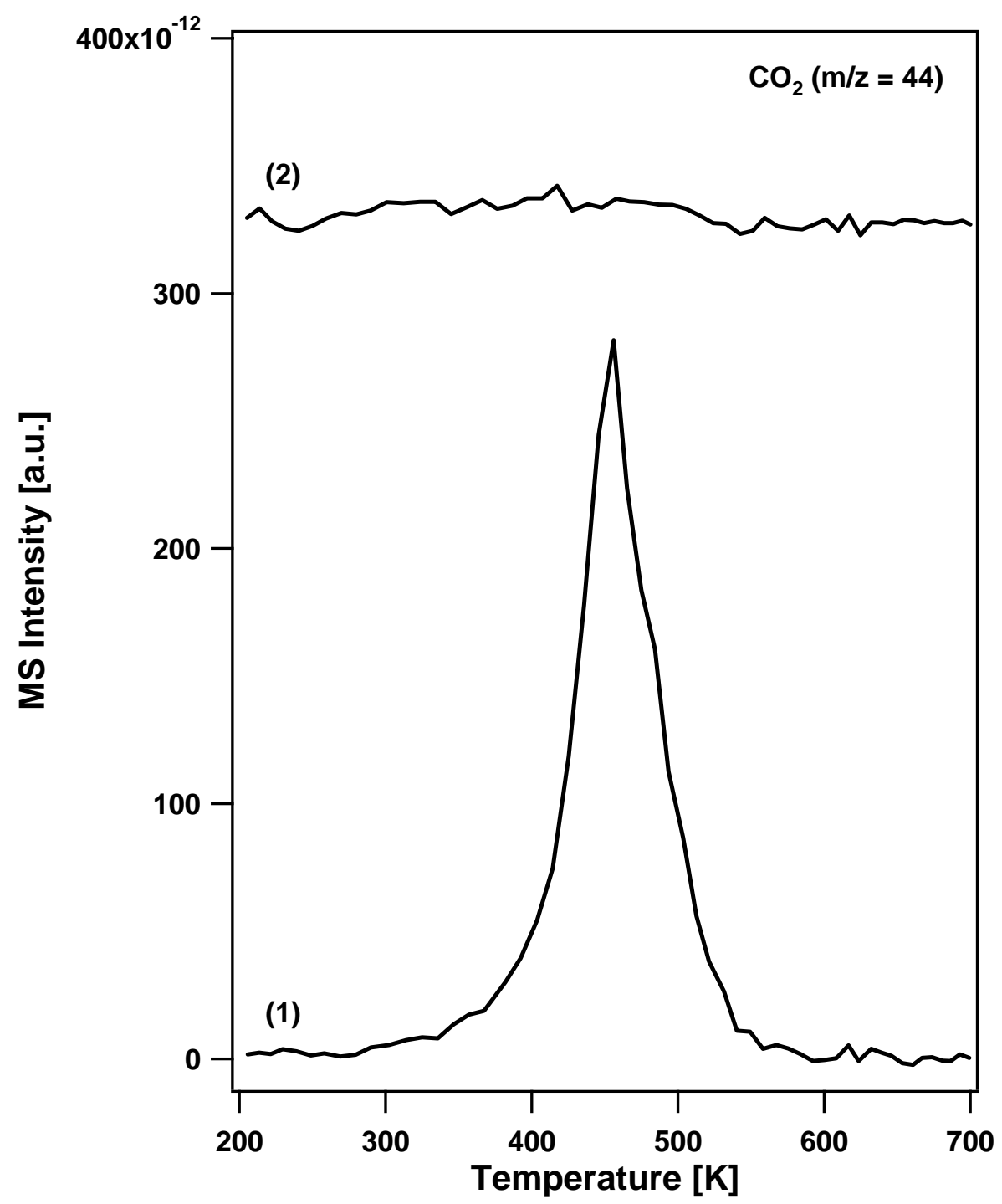

Figure S2. Post-oxidation of (1) the surface after the reaction of styrene with $0.3 \mathrm{ML}$ oxygen-covered $\mathrm{Au}(111)$ and (2) the surface after the reaction of styrene with $\mathrm{Au}(111)$ with 1.2 ML pre-dosed chlorine followed by the same ozone dosage as in (1). Oxygen was deposited on the surface by dosing ozone at $200 \mathrm{~K}$. 
Styrene epoxidation on a very low coverage ( 0.06 ML) of O on $\mathrm{Au}(111)$ at $200 \mathrm{~K}$ :

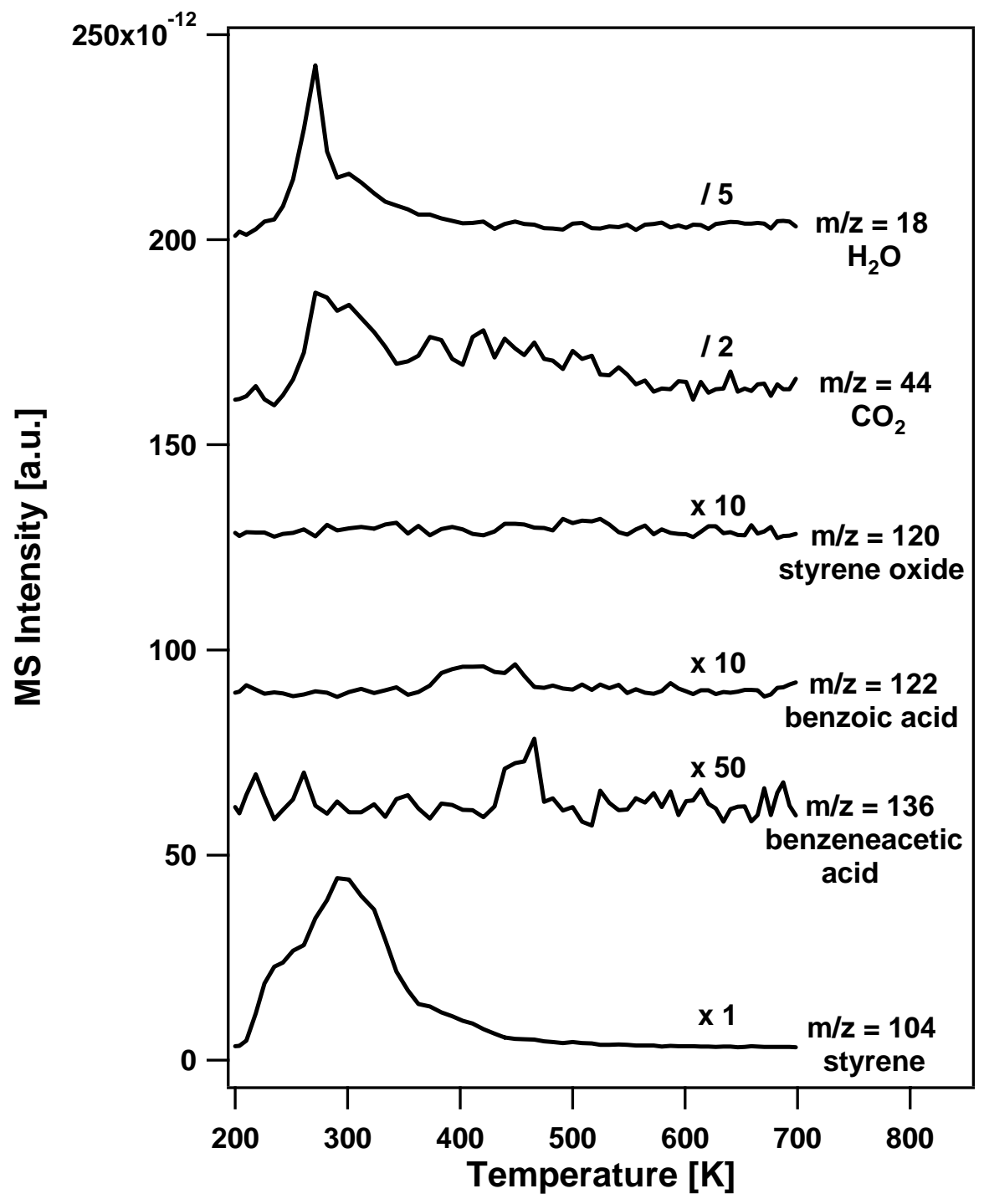

Figure S3. Temperature-programmed reaction of a styrene multilayer on $\sim 0.06$ ML Ocovered $\mathrm{Au}(111)$. Oxygen was deposited on the surface by dosing ozone at $200 \mathrm{~K}$. The styrene adsorption temperature was $200 \mathrm{~K}$. 
TPRS after isothermal reaction of styrene on $\mathrm{O} / \mathrm{Au}(111)$ at $300 \mathrm{~K}$ :

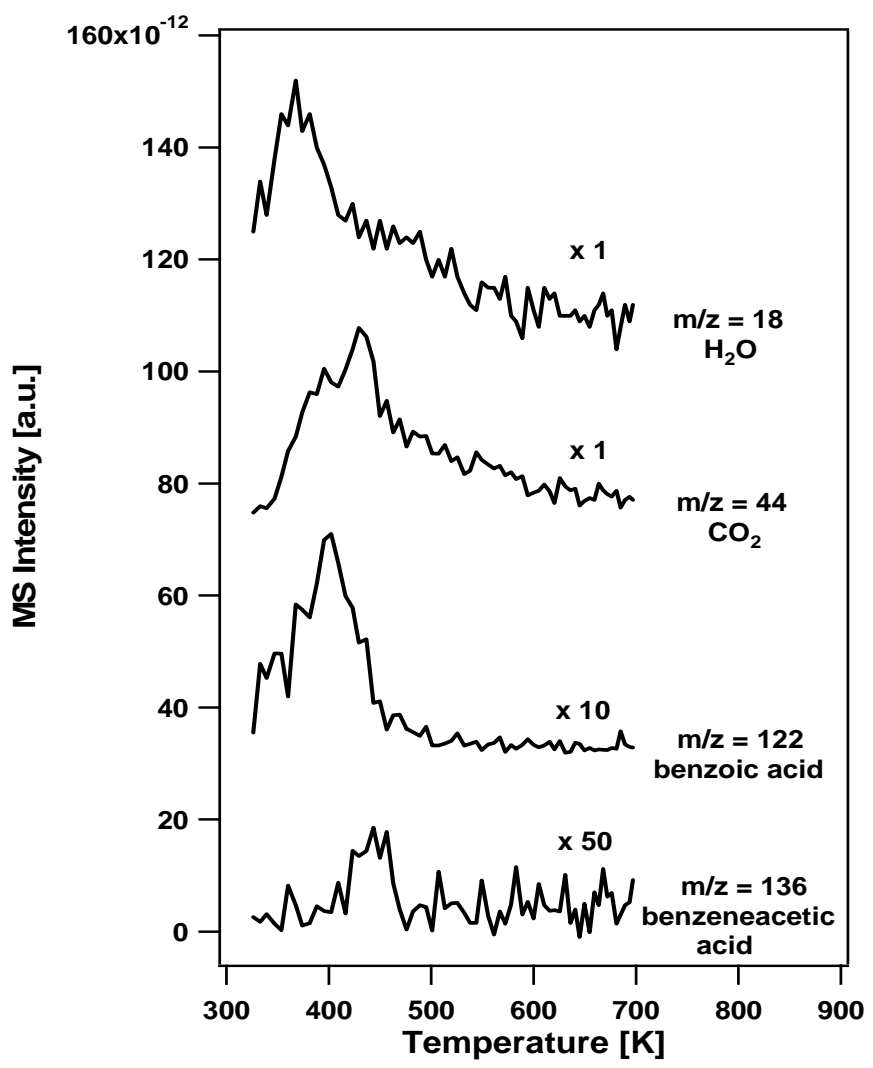

(a)

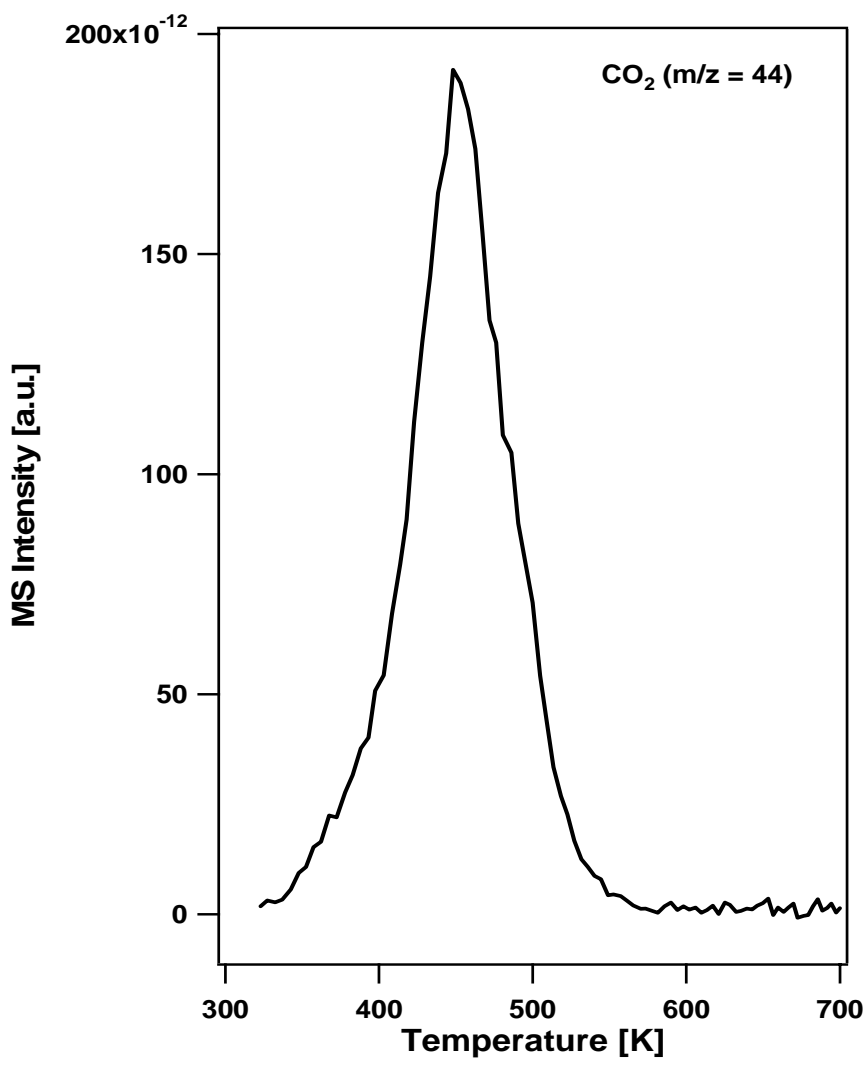

(b)

Figure S4. (a). Temperature-programmed reaction after isothermal reaction of styrene on $0.3 \mathrm{ML} \mathrm{O} / \mathrm{Au}(111)$ at $300 \mathrm{~K}$. (b). Mass $44 \mathrm{TPD}$ from post-oxidation of the surface following (a). 
STM images showing the dispersion of Au-O complexes by chlorine:

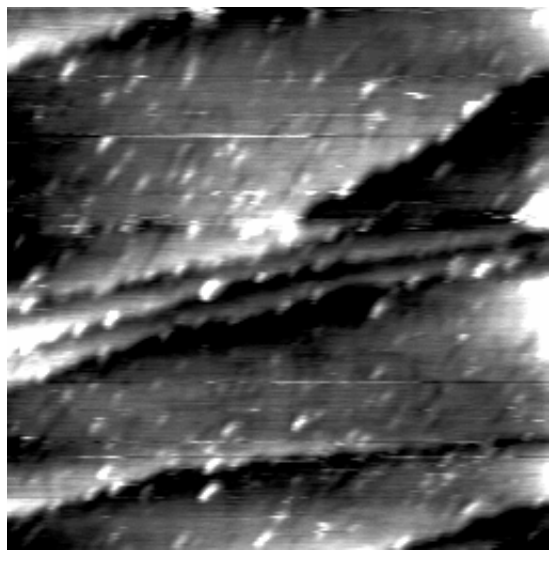

(a)

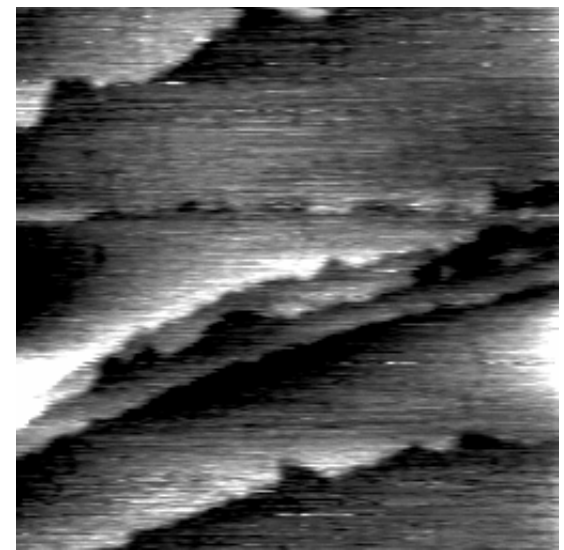

(b)

Figure S5. (a). STM image (70 $\mathrm{nm} \times 70 \mathrm{~nm})$ showing Au-O islands (bright regions) formed by dosing ozone on $\mathrm{Au}(111)$ at $300 \mathrm{~K}\left(\theta_{\mathrm{O}} \sim 0.3 \mathrm{ML}\right)$. (b). STM image (70 $\mathrm{nm} \times 70$ $\mathrm{nm})$ showing the dispersion of Au-O complexes by dosing chlorine ( $\sim 6 \mathrm{~L})$ at $300 \mathrm{~K}$, on the surface shown in (a). Notably, the bright, Au-O islands are absent, indicating dispersal. The streaks seen in image (b) are indicative of the mobile species on the surface. These are preliminary, unpublished results.

\section{References:}

(1) Saliba, N.; Parker, D. H.; Koel, B. E. Surf. Sci. 1998, 410, 270.

(2) Min, B. K.; Alemozafar, A. R.; Biener, M. M.; Biener, J.; Friend, C. M. Top. Catal. 2005, 36, 77.

(3) Kastanas, G. N.; Koel, B. E. Appl. Surf. Sci. 1993, 64, 235. 